\title{
Molecular weight effects on chain pull-out fracture of reinforced polymeric interfaces
}

\author{
Mohsen Sabouri-Ghomi, ${ }^{1}$ Slava Ispolatov, ${ }^{1,2}$ and Martin Grant ${ }^{1}$ \\ ${ }^{1}$ Physics Department and Center for the Physics of Materials, Rutherford Building, McGill University, \\ 3600 rue University, Montréal, Québec, Canada H3A 2 T8 \\ ${ }^{2}$ Baker Laboratory, Chemistry Department, Cornell University, Ithaca, New York 14853
}

(Received 1 April 1999)

\begin{abstract}
Using Brownian dynamics, we simulate the fracture of polymer interfaces reinforced by diblock connector chains. We consider the mushroom regime, where connector chains are grafted with low surface density, for the case of large pulling velocities. We find that for short chains the interface fracture toughness depends linearly on the degree of polymerization $N$ of the connector chains, while for longer chains the dependence becomes $N^{3 / 2}$. Based on the geometry of the initial chain configuration, we propose a scaling argument that accounts for both short and long chain limits and the crossover between them. [S1063-651X(99)10010-2]

PACS number(s): 81.05.Qk, 62.20.Mk, 68.35.Gy
\end{abstract}

Creating advanced materials often means mixing different homopolymers to produce systems with desired combined properties. However, most polymer blends are immiscible: They form macroscopically phase-separated mixtures with only interfacial van der Waals forces, keeping domains of different phases together. The fracture toughness of such blends is limited by one of the interfaces: In ideal defectless conditions, it is equal to the work of adhesion $W=\gamma_{a}+\gamma_{b}$ $-\gamma_{a b}$ between two homopolymer phases. Here, $\gamma_{a}$ and $\gamma_{b}$ are respectively the surface energy of homopolymer $\mathbf{A}$ and homopolymer $\mathbf{B}$ and $\gamma_{a b}$ is the interfacial free energy. Reinforcement of these weak polymeric interfaces is often achieved by the addition of $\mathbf{A}-\mathbf{B}$ diblock copolymers that compatibilize the blend and strengthen the interface [1-3]. The strengthening can be attributed to the miscibility of each block with one of the homopolymers. This causes the block copolymer to expand and entangle with homopolymer phases on either side of the interface. The interfacial tension (energy) is reduced, the interfacial width is increased, and the adhesion thereby improved. Fracture toughness and failure mechanisms of such reinforced polymeric interfaces have been investigated extensively by, for example, experiments on different incompatible systems of polymer glasses [4-10] and cross-linked networks (elastomers) [11,12].

Several theoretical models have been proposed [13-16] to explain the reinforcing effect of connector chains in both elastomers and glassy polymers. A "failure mechanism map" has been developed $[5,15,17,10]$ which relates the mechanism of interface failure to the polymerization index $N$, surface density $\sigma$ of connector chains, and the time scale on which the deformation occurs. According to the failure map there are three major mechanisms: (i) chain scission, which happens whenever the stress along the connector chain becomes larger than the strength of the covalent bond between segments of the chain, (ii) pull-out of the connector chain as a result of disentanglement from homopolymer phase, and (iii) failure by craze formation, followed by chain scission or chain pull-out, which take place when a large stress is transfered to the bulk of the homopolymer phases.

In this paper we direct our attention to the case where interface failure is due to chain pullout, and specifically focus on the effect of the polymerization index $N$ of the con- nector chain on the fracture toughness $G$, the work required to separate the interfaces. We consider a "mushroom regime" [18-23], where connector chains are grafted with low surface density, $\sigma \ll 1 / N l^{2}$ ( $N$ is the number of monomers per grafted chain and $l$ is the monomer size). In this low-density regime, the equilibrium shape of the connector chain in the homopolymer phase is mushroom or plumelike. Furthermore, mutual entanglement between different connectors is negligible. Hence, we need to consider only the behavior of a single chain. Experimental data on the dependence of $G$ on $N$ is scattered, typically assuming a form

$$
G \sim N^{\alpha}
$$

where estimates give variously $1 \leqslant \alpha \leqslant 2[5,10]$. Both linear and quadratic dependences of $G$ on $N$ have been predicted, utilizing a tube picture [24]. Different constitutive equations have been proposed which relate the local stress in planar cohesive zone near the crack tip to various phenomena, such as the penetration depth of the chains, their surface density, and the pulling rate [13-15]. These studies have been focused mainly on the pull-out fracture in tensile mode, when the applied force is normal to the interface. An alternative mode of interface failure is shear fracture, or the resistance of the interface against slip. In fracture mechanics of bulk materials generally only the tensile or opening mode is important, since cracks normally travel in a direction that maximizes the opening mode. However, for the interface between two different materials, the situation can be more complex: The crack is often constrained to follow the interface, giving rise to the possibility of crack propagation involving a combination of tensile and shear modes [2]. Herein we study the dynamics of chain pull-out fracture in both tensile and shear mode separately and examine the dependence of $G$ on $N$.

We use a Brownian dynamics method, due to Pickett, Jasnow, and Balazs [18], which was adapted and modified by those authors from a model of DNA in gel electrophoresis [25], to simulate the pullout of a single connector chain of length $N$ from a two-dimensional homopolymer phase. To quantify the interface toughness for different chain length, we calculate the work that is required to pull out the chain with constant velocity $\mathbf{v}_{\mathbf{0}}$. The homopolymer phase is modeled by a two-dimensional semi-infinite square lattice [26] of 
obstacles. Each obstacle represents an entanglement or crosslinked point, depending on the glassy or elastomeric structure of the homopolymer phase, and provides lateral constraint on the movement of the connector chain. The connector is represented by a freely jointed chain of $N$ beads connected by rigid links, with no self-interaction between the beads other than the constraint of constant segment length. The initial configuration is created by putting the first monomer at the boundary and then continuing the chain as a random walk in dimension $d=2$, with a reflecting boundary at the interface. The random walk is restricted by requiring, as we shall see below, that monomers are repelled by the obstacles. The pull-out dynamics is simulated by pulling the chain by the first monomer at a constant velocity in either tensile mode (perpendicular to the interface) or shear mode (parallel to the interface). This is done conveniently by moving the obstacle matrix at velocity $+\mathbf{v}_{\mathbf{0}}$ while keeping the first monomer fixed. That is, if $\mathbf{r}_{\mathbf{i}}$ is the position of the $i$ th monomer, $d \mathbf{r}_{1} / d t \equiv 0$.

The movement of the other $i=2, \ldots, N$ monomers is governed by the following over-damped Langevin equation:

$$
\nu\left(\frac{d \mathbf{r}_{\mathbf{i}}}{d t}-\mathbf{v}_{\mathbf{0}}\right)=\tau_{i}\left(\mathbf{r}_{\mathbf{i}+\mathbf{1}}-\mathbf{r}_{\mathbf{i}}\right)-\tau_{i-1}\left(\mathbf{r}_{\mathbf{i}}-\mathbf{r}_{\mathbf{i}-1}\right)+\mathbf{F}_{\mathbf{i}}+\boldsymbol{\eta}_{\mathbf{i}}
$$

where $\mathbf{r}_{\mathbf{N}+\mathbf{1}} \equiv \mathbf{r}_{\mathbf{N}}$. Here, $\tau_{i}$ is the amplitude of tension in the segment connecting monomers $i$ and $i+1, \boldsymbol{\eta}_{\mathrm{i}}$ is the random Gaussian noise representing the effect of thermal fluctuations on each monomer, and $\nu$ is the viscous friction coefficient per monomer. The strength of the vectorial random noise is related to the monomeric friction by the fluctuationdissipation theorem for its components, labeled by Greek superscripts, $\left\langle\eta_{i}^{\beta}(t) \eta_{j}^{\beta^{\prime}}\left(t^{\prime}\right)\right\rangle=2 \nu k_{B} T \delta_{\beta, \beta^{\prime}} \delta_{i, j} \delta\left(t-t^{\prime}\right)$, where $k_{B}$ is Boltzmann's constant and $T$ is temperature. $\mathbf{F}_{i}\left(\Delta \mathbf{r}_{\mathbf{i}}\right)$ is a short range monomer-obstacle repulsive force, where $\Delta \mathbf{r}_{\mathbf{i}}$ is the distance between the $i$ th monomer and the closest obstacle. For $\left|\Delta \mathbf{r}_{\mathbf{i}}\right|<r_{c}$, there is hard-core repulsion. For $r_{c}<\left|\Delta \mathbf{r}_{\mathbf{i}}\right|<2 r_{c}$, there is soft-core repulsion, where the force obeys

$$
\mathbf{F}_{\mathbf{i}}=\sigma \Delta \mathbf{r}_{\mathbf{i}}\left(\frac{1}{\left|\Delta \mathbf{r}_{\mathbf{i}}\right|^{2}-r_{c}^{2}}-\frac{1}{3 r_{c}^{2}}\right)^{2}
$$

The parameter $\sigma$ controls the strength of the force [25]. For larger separations, $\left|\Delta \mathbf{r}_{\mathbf{i}}\right|>2 r_{c}$, there is no force acting on the monomer. In our simulations we have used $r_{c}=l / 2$, where $l$ is the link length of the connector chain.

To solve Eq. (2), the tensions $\tau_{i}$ in each segment of the connector chain have to be determined. This is done by enforcing the constant segmental length constraint,

$$
\left|\mathbf{r}_{\mathbf{i}+\mathbf{1}}-\mathbf{r}_{\mathbf{i}}\right|^{2}=l^{2}
$$

for $1<i<N-1$. Taking the derivative of this with respect to time, and using Eq. (2), results in a tridiagonal matrix equation for the $\tau_{i}$ 's [25],

$$
\begin{aligned}
\nu \frac{d\left(\mathbf{r}_{\mathbf{i}+\mathbf{1}}-\mathbf{r}_{\mathbf{i}}\right)^{2}}{2 d t}= & \tau_{i+1}\left(\mathbf{r}_{\mathbf{i}+\mathbf{2}}-\mathbf{r}_{\mathbf{i}+\mathbf{1}}\right) \cdot\left(\mathbf{r}_{\mathbf{i}+\mathbf{1}}-\mathbf{r}_{\mathbf{i}}\right)-2 \tau_{i} l^{2} \\
& +\tau_{i-1}\left(\mathbf{r}_{\mathbf{i}}-\mathbf{r}_{\mathbf{i}-\mathbf{1}}\right) \cdot\left(\mathbf{r}_{\mathbf{i}+\mathbf{1}}-\mathbf{r}_{\mathbf{i}}\right)+\left(\mathbf{F}_{\mathbf{i}+\mathbf{1}}+\boldsymbol{\eta}_{i+1}\right. \\
& \left.-\mathbf{F}_{\mathbf{i}}-\boldsymbol{\eta}_{\mathbf{i}}\right) \cdot\left(\mathbf{r}_{\mathbf{i}+\mathbf{1}}-\mathbf{r}_{\mathbf{i}}\right) .
\end{aligned}
$$

Of course, application of the constant segmental length constraint implies that the left-hand side of Eq. (5) should be zero. However, after several updates of the simulation, an accumulation of roundoff errors causes the distances between some of the adjacent monomers to differ slightly from $l$. Therefore, we have used a correcting factor to ensure the segmental length stays fixed, and that the algorithm is stable,

$$
\frac{d\left(\mathbf{r}_{\mathbf{i}+\mathbf{1}}-\mathbf{r}_{\mathbf{i}}\right)^{2}}{2 d t}=-\left|\mathbf{r}_{\mathbf{i}+\mathbf{1}}-\mathbf{r}_{\mathbf{i}}\right| \frac{\left(\left|\mathbf{r}_{\mathbf{i}+\mathbf{1}}-\mathbf{r}_{\mathbf{i}}\right|-l\right)}{d t} \text {. }
$$

Equation (2) is solved using a fourth-order Runga-Kutta algorithm.

We consider reduced units in which $\nu, \mathrm{v}_{0}, \sigma, k_{B} T$, and $D$, the distance between obstacles, are set equal to unity. The distance between two adjacent monomers in connector chain $l$ is chosen to be 0.4 . The time step of the simulation, typically $10^{-4}$ to $10^{-5}$, is adjusted so that the average difference in segment length from $l$ in each run is less than $0.1 \%$. The results were averaged over $20-100$ independent realizations of the initial conditions.

Figure 1 shows a snapshot of the simulation for both tensile and shear pullout. We quantify the fracture toughness of the interface by determining the work $G$ required to remove the connector chain completely from the obstacle lattice. The work is $G=\int P d t$, where the power $P$ is obtained via $P$ $=\mathrm{v}_{0} \tau, \mathrm{v}_{0}$ is the constant pulling velocity, and $\tau$ is the instantaneous tension in the segment crossing the boundary of the obstacle lattice in the pulling direction at each time step. In the case of the shear mode, the boundary being crossed is indicated in Fig. 1(b).

Figure 2 shows the fracture toughness $G$ as a function of the polymerization index $N$ of the connector chain for tensile and shear modes. Naturally, the fracture toughness grows as the degree of polymerization of the connector chain increases. This growth is more significant for larger $N$ due to the fact that longer connectors can penetrate well beyond the neighborhood of the interface. They entangle efficiently with the bulk polymers of the compatible phase. It also shows that the effect of reinforcement on the interface is higher in tensile mode, by a factor of about 5 for the present model [27]. One can notice the existence of two scaling regimes for $G$ versus $N$, corresponding to short connectors, $N<N_{c}$, and long connectors, $N>N_{c}$, where $N_{c}$ is the crossover length. Figure 3 shows the power-law dependence of $G$ on $N$. For short connectors $G$ scales linearly with $N, G \sim N$, while for long connectors it scales as $G \sim N^{3 / 2}$.

These regimes can be understood as follows. For small $N$, the chain is entangled with one or two layers of obstacles, even if the free radius of gyration $R_{g} \approx l \sqrt{N}$ is of order or smaller than the obstacle lattice spacing $D$. Since the the initial configuration is repelled by the obstacles, the available phase space for a chain is quite restricted. For the first few chain segments, the reflecting boundary condition and the the area taken up by the obstacles results in the chains going 


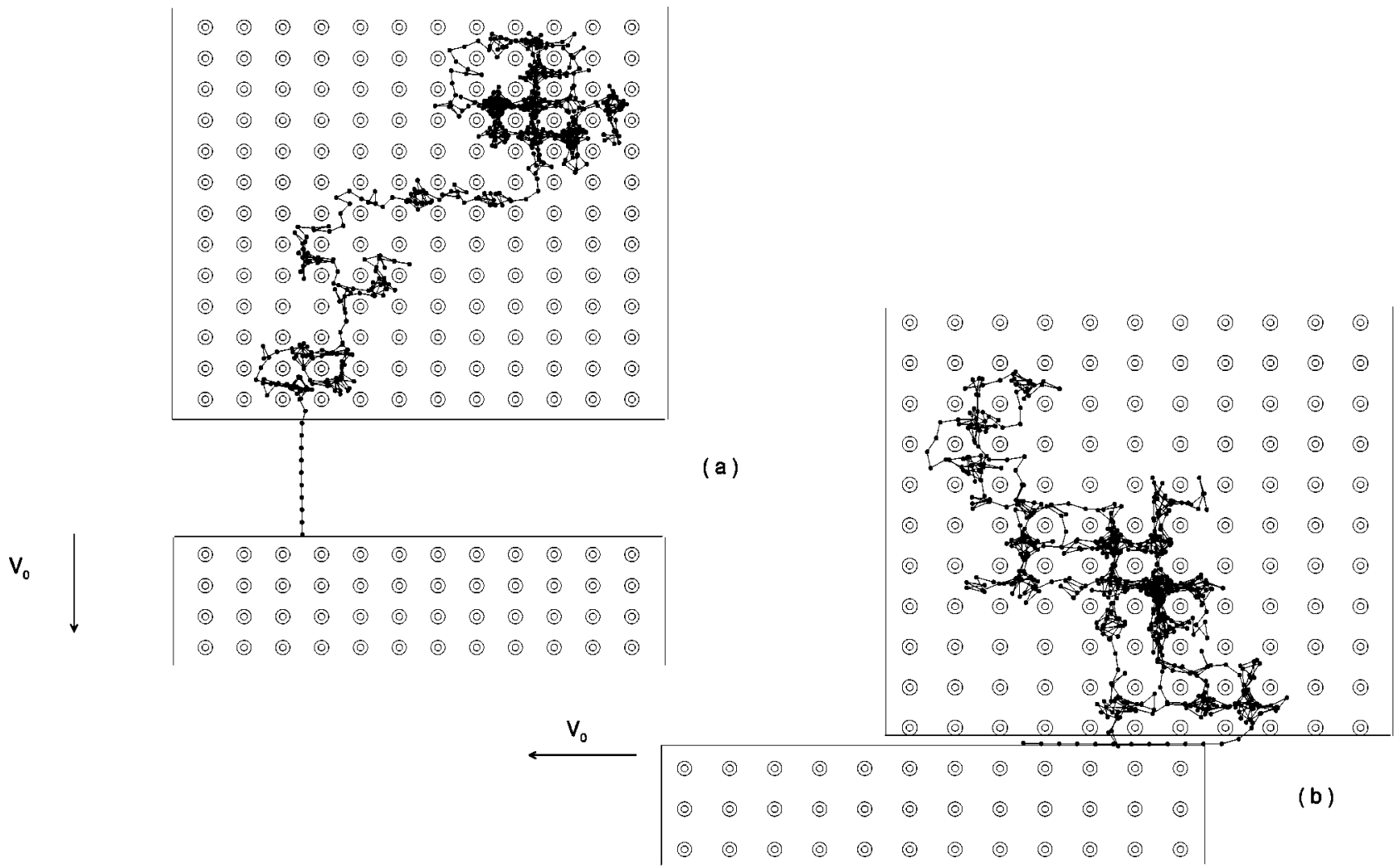

FIG. 1. Snapshot of a simulation of fracture for a chain of $N=700$ monomers: (a) tensile and (b) shear mode.

straight into the lattice. After passing the first row or two of obstacles, the chain, if it is long enough, usually bends, with tight loops around obstacle cores being highly improbable. When the chain is pulled out, the dominant deterministic part of the tension equals the total viscous drag force. This force is roughly proportional to the number of monomers $N_{\text {move }}$ simultaneously in motion. Since pulling tension cannot propagate through a loop unless it is tightened around an obstacle core, for short chains $N_{\text {move }}$ is of the order of the number of monomers stretched in one obstacle lattice spacing $D$, that is, $D / l$. Hence for small $N$, the total work to pull the chain out can be estimated as

$$
G=\mathrm{v}_{0} \int_{0}^{N l / \mathrm{v}_{0}} F_{d r a g} d t \sim \mathrm{v}_{0}^{2} \nu \frac{D}{l} \int_{0}^{N l / \mathrm{v}_{0}} d t=\mathrm{v}_{0} \nu N D,
$$

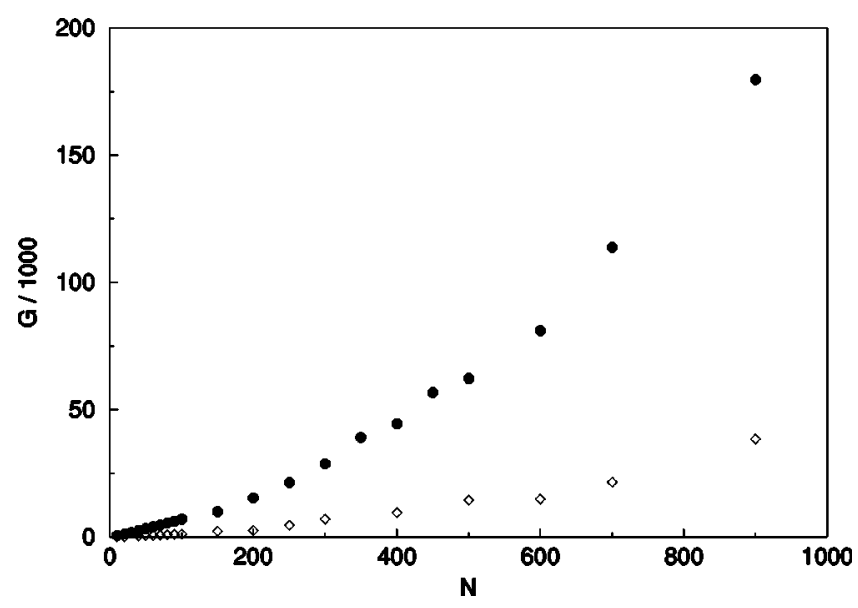

FIG. 2. Fracture toughness $G$ vs $N$ polymerization index of the connector chain in tensile $(\bigcirc)$ and shear $(\diamond) . G$ is dimensionless. where the integral is over the time it takes to remove the chain from the matrix, which is consistent with the linear scaling regime observed in simulations for short connectors.

For large $N$, the penetration depth increases. Indeed, for sufficiently large $N$, the chain center of mass is located at a distance of the order of the radius of gyration $R_{g}$ from the interface. Then, the number of monomers that are simultaneously in motion becomes proportional to this distance, $N_{\text {move }} \approx R_{g} \sim N^{1 / 2}$, which we have observed directly. Consequently, the pull-out fracture energy becomes

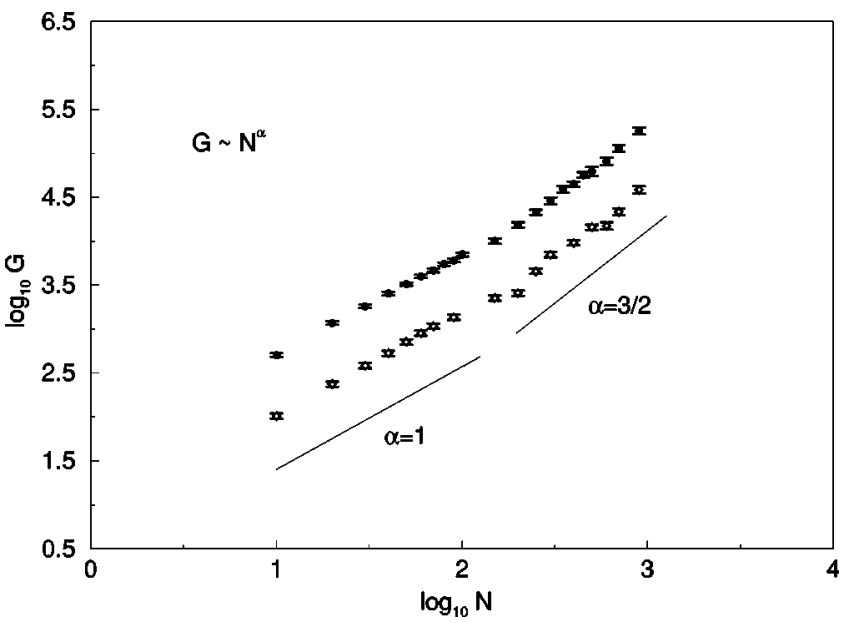

FIG. 3. $\log _{10}-\log _{10}$ plot of $G$ vs $N$. (O) represents tensile and $(\diamond)$ indicates shear mode. Solid lines show that short-chain data $(N \leqslant 100)$ are consistent with $\alpha=1$, while long-chain data $(N$ $>100$ ) are consistent with $\alpha=3 / 2$. $G$ is dimensionless. 


$$
G=\mathrm{v}_{0} \int_{0}^{N l / \mathrm{v}_{0}} F_{\text {drag }} d t \sim \mathrm{v}_{0}^{2} \nu \int_{0}^{N l / \mathrm{v}_{0}} N^{1 / 2} d t=\mathrm{v}_{0} \nu N^{3 / 2} l,
$$

where the integral is over the time it takes to remove the chain from the matrix. This is roughly analogous to what happens when one pulls on a wet garden hose of length $N$, left on the ground by a gardener who has performed a random walk among a grove of trees. Upon pulling, only the currently stretched segment starts to move, if friction in the self-intersections of the hose is negligible. If the hose configuration was created as a result of a random walk, the length of such a segment scales as a typical size of such a walk, $\sqrt{N}$, where $N$ is the total length of the hose. The hose configuration may include a few loops around the tree trunks; upon tightening, these loops also start to move, assuming friction is negligible. The total length of these tightened loops has no important contribution: That length scales as a winding angle of a random walk of the length $N$, i.e., as $\ln N$ (see, for example, Ref. [28]). As a result, the drag force is proportional to the length of the hose that is constantly in motion, $\sqrt{N}$.

Although our numerical work is in two dimensions, these physical arguments also follow in three dimensions, and are applicable to experimental systems [29]. The small $N$ regime crosses over to a large $N$ regime when the radius of gyration $R_{g}$ becomes of order of the obstacle spacing $D$. That gives $N_{\text {cross }} \sim[D / l]^{2}$, and we expect

$$
G=N f\left(N / N_{\text {cross }}\right),
$$

where the crossover scaling function obeys $f(x \rightarrow 0)$ $=$ const, and $f(x \rightarrow \infty)=x^{1 / 2}$.

As mentioned earlier, different forms of the power-low dependence of $G$ on $N$ have been proposed in previous theoretical studies: Both $G \sim N$ and $G \sim N^{2}$ have been predicted. The results from experiments are somewhat ambiguous. The linear dependence of $G$ on $N$ [13] is predicted for very slow crack propagation velocities, and therefore corresponds to $G \rightarrow G_{0}$, where $G_{0}$ is the fracture toughness threshold or minimal energy required to break the interface when $v_{0}$ $\rightarrow 0$. On the other hand, $G \sim N^{2}$ is predicted for pull-out fractures where the crack propagation speed is high or at least larger than a critical velocity. In other words, these two predicted regimes differ by the pull-out velocity. The crossover between the two scaling regimes $G \sim N$ and $G \sim N^{3 / 2}$ obtained in our simulations takes place in the latter high velocity regime: The pulling velocity used in our simulations $\mathrm{v}_{0}$ $=k_{B} T /(\nu D)$ corresponds to relatively fast pulling rate [30], which in turn results in high crack propagation speeds. Our work is in the same regime, and indeed extends the numerical work and theoretical arguments of Pickett, Jasnow, and Balazs [18], wherein this model was introduced. For large $N$, Picket et al. argued $G \sim N^{2}$ in contrast to our result of $G$ $\sim N^{3 / 2}$. This was based on an analogy of the polymer to the motion of a rope in "block and tackle" pulleys. In that case, the average drag force to pull the connector chain out of the matrix is $f_{\text {drag }} \sim \nu \mathrm{V}_{0} N$. Hence, it follows that the number of monomers instantaneously in motion are $N_{\text {move }} \sim N$. In fact, the correct analogy is not to pulleys, but to the motion of a garden hose lying on grass, as described above. Then $f_{\text {drag }}$ $\sim N^{1 / 2}$, giving rise to the $3 / 2$ exponent. Our numerical results, which are more extensive than those of the earlier work, support this picture.

To conclude, we studied the chain pull-out fracture of a reinforced polymeric interface in tensile and shear modes. Our results confirm the nonlinear dependence of the fracture toughness of the interface on the length of the connector chain observed in experiments. We found that, depending on the length of connector chain, the fracture toughness of the interface shows different scaling dependences: For short chains, $G$ scales linearly with $N$, while for long connectors, we observed a crossover to a new scaling regime $G \sim N^{3 / 2}$. Our results can be tested experimentally on elastomeric networks for long connector chains in the low coverage mushroom regime [31].

This work was supported by the Natural Sciences and Engineering Research Council of Canada and le Fonds pour la Formation de Chercheurs et l'Aide à la Recherche de Québec.
[1] D. R. Paul, in Polymer Blends and Alloys, edited by D. R. Paul and S. Newman (Academic, New York, 1978), Vol. 2; H. Brown, Annu. Rev. Mater. Sci. 21, 463 (1991); H. R. Brown, K. Char, V. R. Deline, and P. F. Green, Macromolecules 26, 4155 (1993).

[2] H. Brown, Annu. Rev. Mater. Sci. 21, 463 (1991).

[3] H. R. Brown, Mater. Sci. Forum 189-190, 57 (1995).

[4] H. R. Brown, Macromolecules 22, 2859 (1989).

[5] C. Creton, E. J. Kramer, C.-Y. Hui, and H. R. Brown, Macromolecules 25, 3075 (1992).

[6] J. Washiyama, C. Creton, E. J. Kramer, F. Xia, and C.-Y. Hui, Macromolecules 26, 6011 (1993).

[7] J. Washiyama, E. J. Kramer, and C.-Y. Hui, Macromolecules 26, 2928 (1993).

[8] F. Xiao, C. Y. Hui, J. Washiyama, and E. J. Kramer, Macromolecules 27, 4382 (1994).

[9] C.-A. Dai, B. J. Dair, K. H. Dai, C. K. Ober, E. J. Kramer,
C.-Y. Hui, and L. W. Jelinski, Phys. Rev. Lett. 74, 2837 (1995).

[10] J. Washiyama, E. J. Kramer, C. F. Creton, and C.-Y. Hui, Macromolecules 27, 2019 (1994).

[11] H. R. Brown, Macromolecules 26, 1666 (1993).

[12] C. Creton, H. R. Brown, and K. R. Shull, Macromolecules 27, 3174 (1994).

[13] E. Raphaël and P. G. de Gennes, J. Phys. Chem. 96, 4002 (1992); H. R. Brown, C.-Y. Hui, and E. Raphaël, Macromolecules 27, 608 (1994).

[14] H. Ji and P. G. de Gennes, Macromolecules 26, 520 (1993).

[15] D. B. Xu, C.-Y. Hui, E. J. Kramer, and C. Creton, Mech. Mater. 11, 257 (1991).

[16] K. E. Evans, J. Polym. Sci., Part B: Polym. Phys. 25, 353 (1987); B. Lin and P. L. Taylor, Macromolecules 27, 4212 (1994); K. P. O'Connor and T. C. B. McLeish, Polymer 33, 4314 (1992); P. Prentice, ibid. 24, 346 (1983); M. Rubenstein, 
Polym. Prepr. (Am. Chem. Soc. Div. Polym. Chem.) 53, 628 (1994).

[17] C. Y. Hui, A. Ruina, C. Creton, and E. J. Kramer, Macromolecules 25, 3948 (1992); H. R. Brown, ibid. 24, 2752 (1991).

[18] G. T. Pickett, D. Jasnow, and A. C. Balazs, Phys. Rev. Lett. 77, 671 (1996).

[19] J. B. Field, C. Toprakcioglu, L. Dai, G. Hadziioannou, G. Smith, and W. Hamilton, J. Phys. France II 2, 2221 (1992).

[20] H. Motschmann, M. Stamm, and C. Toprakcioglu, Macromolecules 24, 3681 (1991).

[21] A. T. Clark and M. Lal, J. Chem. Soc., Faraday Trans. 2 74, 1857 (1978).

[22] K. P. O'Connor and T. C. B. McLeish, Macromolecules 26, 7322 (1993); J. M. Deutsch and H. Yoon, ibid. 27, 5720 (1994).

[23] The degree of the coverage of the interface by the grafting chains plays an important role. Neutron diffraction measurements [19] and adsorption kinetic studies [20] together with Monte Carlo simulations [21] of configurational properties of grafted diblock copolymers reveal a crossover from essentially unperturbed "mushroom" conformation at low surface coverage to strongly stretched "brush" conformation at high surface coverage. In our simulations we consider the low coverage regime where the connector chain takes a nonbiased random mushroomlike configuration. It is consistent with the observed equilibrium configuration of a single chain grafted to a reflective wall in contact with an elastomeric network $[22,19]$.

[24] P. G. de Gennes, Scaling Concepts in Polymer Physics (Cornell University Press, Ithaca, 1979); M. Doi and S. F. Edwards, The Theory of Polymer Dynamics (Clarendon Press, Oxford, 1986).

[25] J. M. Deutsch, Science 240, 922 (1988); J. M. Deutsch and T. L. Madden, J. Chem. Phys. 90, 2476 (1989).

[26] We ran sample simulations involving a random obstacle matrix to ensure that the regularity of the obstacle matrix had no measurable effect on our results.
[27] Using different values for the thermal noise in our simulations, we confirmed numerically that the primary origin of the differences in fracture toughness in shear and tensile modes was thermal. In the shear mode, the crossing bond is always anchored by one of the obstacles in the boundary of the obstacle lattice. Therefore, most of the thermal noise force on the crossing bond is absorbed by the soft potential shell around the anchoring obstacle. This results in a reduction of the tension in the crossing bond and hence, in the fracture toughness.

[28] B. Drossel and M. Kardar, Phys. Rev. E 53, 5861 (1996).

[29] If the initial configuration is created including a small bias in the random walk, then we expect that, for large $N$, the configuration size in the direction of the bias will become proportional to $N$ itself, giving $G \sim N^{2}$.

[30] Other than computational expense, two further considerations prevented us from simulating the slow velocity regime. First, at low velocity, the rigid obstacle will be a poor representation of the moving polymer surrounding, because of the possibility of significant deformation in the matrix during the pull-out period. Second, at very low velocities, there may be a different form for friction than the monomeric form we have used, e.g., friction may take a form similar to that used to describe the arm retraction of star polymers: see A. Ajdari, F. BrochardWyart, P. G. de Gennes, L. Leibler, J. L. Viovy, and M. Rubinstein, Physica A 204, 17 (1994).

[31] Experimental measurements of fracture toughness of reinforced glassy and elastomeric polymer interfaces by diblock copolymers have been carried for connector chains with very high polymerization index ( $N$ up to 870 for glassy [5] and $N$ up to 2205 for elastomeric [12] interfaces). Nevertheless, there are only two sets of experimental data available $[5,10]$ on the power law dependence of fracture toughness $G$ on polymerization index $N$. These are obtained from experiments on glassy interfaces for connector chains of $N \leqslant 270$ with brushy initial configurations. Hence, those experiments cannot provide a test of our results. 